\title{
GI highlights from the literature
}

\author{
Philip J Smith
}

\section{BASIC SCIENCE}

\section{Targeting Notch to prevent inflammation-related fibrosis in non-alcoholic steatohepatitis (NASH)}

Yu J, Zhu C, Wang X, et al. Hepatocyte TLR4 triggers interhepatocyte Jagged1/Notch signalling to determine NASH-induced fibrosis. Sci Transl Med 2021; 13599: eabe1692. doi: 10.1126/

scitranslmed.abe1692.

NASH remains an enormous clinical problem with limited medical therapies to prevent end-stage liver disease and cancer. Emerging data have suggested a role in the so-called Notch intercellular signalling pathway in NASH-related fibrosis. To examine this further, Yu et al combined analysis of human tissue and mechanistic preclinical mouse studies. First, by retrospectively examining clinical trial data, they observed that the Notch ligand Jagged 1 (Jag1) was the only Notch pathway component that differed between those who responded to trial therapy (pioglitazone or vitamin E) versus non-responders, an observation supported by an independent NASH cohort. In a mouse model, they confirmed zonal hepatocellular Jag1 expression with dietary induced NASH. Using hepatocytespecific knockout of the Jag1 gene, they prevented juxtaposed hepatocytes activating the Notch pathway, reducing both stellate cell activation and fibrosis deposition. Conversely, overexpressing Jag1 in hepatocytes resulted in increased fibrosis once mice were fed a NASH-inducing diet. Using a Jag1 promoter reporter, they showed that inflammatory signalling from nuclear factor kappalight-chain-enhancer of activated B cells and Toll-like receptor 4 directly activated this process. Translational therapy, using an antisense oligonucleotide to prevent Jag1 activity in hepatocytes, was tested in mice. This was not only well tolerated and reduced Jag1 and Notch-pathway activation but also reduced fibrosis both in early and established disease. It remains unclear if these studies in shortterm preclinical models will be translatable to human and whether they will impact on cirrhotic disease and other complications such as hepatocellular carcinoma but they provide an exciting step towards a novel therapy for both NASH-disease treatment and prevention.

\section{From nucleotide-binding oligomerisation domain 2 (NOD2) genetic association to molecular function in Crohn's disease} Nayar S, Morrison J, Giri M, et al. A myeloid-stromal niche and gp130 rescue in NOD2-driven Crohn's disease. Nature 2021; 593 (7858): 275-81. doi: 10.1038/s41586-021-03484-5.

Mutations of the NOD2 gene confer the strongest genetic risk for Crohn's disease and associate with ileal stricturing (fibrosis). Fibrosis in Crohn's disease is driven by crosstalk between macrophages (a type of myeloid immune cell) that can differentiate to fibroblasts (a type of stromal cell). Both cell types are linked with anti-tumour necrosis factor (TNF) therapy resistance. Despite these associations, NOD2 mutations have not been linked to anti-TNF response. There is also an apparent contradiction that while NOD2 activation can induce a powerful proinflammatory response, Crohn's polymorphisms identify loss of function, which may be hypothesised as protective rather than deleterious. Unravelling this paradox, Nayar et al demonstrated human ileal resections contain high numbers of activated macrophages and fibroblasts. Zebrafish with NOD2 mutations also demonstrated higher numbers of these cells in the ileum following response to inflammatory stimuli (dextran sulfate sodium) and these cells were also enriched in children carrying NOD2 mutations recently diagnosed with Crohn's. Peripheral blood monocytes from NOD2 mutant subjects had an enhanced ability to differentiate to macrophages and subsequently fibroblasts. STAT3 signalling was upregulated and associated with proinflammatory/profibrotic cytokines interleukin 6 (IL-6), oncostatin M and IL-11, which in turn were associated with anti-TNF resistance. Blockade of glycoprotein 130 (the common cytokine receptor subunit associated with STAT3 activation) using bazedoxifene lessened fibroblast differentiation and reduced intestinal inflammation and damage in the NOD2 mutant zebrafish model. These insights link genetic association of NOD2 loss of function to macrophage-fibroblast function and highlight a potential adjunctive treatment pathway to anti-TNF worthy of further investigation.

\section{Molecular phenotyping in Barrett's oesophagus}

Nowicki-Osuch K, Zhuang L, Jammula S, et al. Molecular phenotyping reveals the identity of Barrett's oesophagus and its malignant transition. Science 2021; 373 (6556): 760-67. doi: 10.1126/science.abd1449.

The origin of Barrett's oesophagus (BE) has been the source of much debate. This has hampered our ability to understand the evolution of the condition to cancer. Recent years have seen the stomach and submucosal gland (SMG) theories becoming more prominent. Nowicki-Osuch et al used a variety of gene expression, open chromatin and wholegenome sequencing to show the relationship of the gastric cardia with BE. The authors used stereomicroscopy and single-cell RNA sequencing (ScRNAseq) analysis to characterise SMGs showing the presence of keratin $7+$ population of cells that were similar to those found at the gastro-oesophageal junction possibly indicating cells that share the same origin. ScRNAseq of areas of BE comparing with normal GI tissues and were able to confirm previous studies that $\mathrm{BE}$ has a similar gene expression to normal gastric tissue but little relationship to SMGs. Interestingly, the authors produced an assay for transposase-accessible chromatin using sequencing data that showed a similar open chromatic landscape to the gastric cardia. Shared mutations between $\mathrm{BE}$ and stomach were detected adding to the evidence that $\mathrm{BE}$ and stomach share a common ancestor. To determine the molecular process of conversion from normal stomach to BE, the authors identified a set of genes differentially expressed in BE versus stomach that included cellular myelocytomatosis and hepatocyte nuclear factor 4 alpha (HNF4A). Organoids transfected with HNF4A expressed a similar gene expression to BE. Additionally, deconvolution of bulk oesophageal adenocarcinoma (OAC) RNA sequencing data showed that BE has related gene expression to OAC. Overall, this is an important paper that shows in more detail the relationship of $\mathrm{BE}$ with surrounding tissue and cancer.

\section{CLINICAL PRACTICE}

\section{Transglutaminase 2 inhibition in coeliac disease}

Schuppan D, Mäki M, Lundin K, et al. A randomized trial of a transglutaminase 2 inhibitor for coeliac disease. N Engl J Med $2021 ; 385$ (1): 35-45.

In the therapy of coeliac disease, there is the dire need for the development of an adjunct treatment to gluten-free diet with capability to significantly induce mucosal healing. Schuppan et al tested efficacy and safety of the oral selective transglutaminase 2 inhibitor ZED1227 in a phase II, double-blind, placebo-controlled trial in patients with well-controlled coeliac disease who were challenged with a moderate amount $(3 \mathrm{~g})$ of daily gluten intake. All applied 
doses of ZED1227 attenuated the gluten-induced small intestinal damage $(p<0.001)$. The estimated difference from placebo $(n=30$ patients with adequate duodenal biopsy samples) in the change in the mean ratio of villus height to crypt depth from baseline to week 6 was 0.44 in the $10 \mathrm{mg}(\mathrm{n}=35), 0.49$ in the $50 \mathrm{mg}(\mathrm{n}=39)$ and 0.48 in the $100 \mathrm{mg}$ group $(\mathrm{n}=38)$. Treatment with ZED1227 dose dependently decreased intraepithelial lymphocyte density in comparison to placebo. Treatment with ZED1227 seemed to decrease the incidence of coeliac disease-associated symptoms and to increase quality of life. With the exception of rash $(n=3$ in the $100 \mathrm{mg}$ group), no adverse events appeared to be more common in the ZED1227 than in the placebo group. This proof of concept study demonstrated for the first time that a non-dietary treatment is able to attenuate gluten-induced damage in the duodenal mucosa of coeliac disease patients. Further studies are needed to investigate the efficacy and safety of ZED1227 in patients with coeliac disease.

\section{Effect of oral ranitidine on urinary excretion of $\mathrm{N}$-nitrosodimethylamine (NDMA)}

Florian J, Matta M, DePalma R, et al. Effect of oral ranitidine on urinary excretion of N-nitrosodimethylamine (NDMA): a randomized clinical trial. JAMA 2021; 326 (3): 240-49.

There has been a major recall of ranitidine due to the potential for increased levels (>96 ng/day) of NDMA which is a carcinogen when ingested over a long period of time. The primary evidence arose from a small study showing an approximate 400 -fold increase in NDMA but there were limitations including no randomisation, no control of environmental or dietary NMDA and few details concerning analytical methods. Florian et al conducted a randomised study comprising 18 individuals with a range of age, sex and ethnicities. They were randomised to receive one of four regimens, $300 \mathrm{mg}$ ranitidine or placebo and also had dietary control for nitrate intake specifying cured or non-cured meats. Blood and urine samples were collected regularly throughout the day following treatment allocation and tested for NDMA, dimethylamine (DMA) and ranitidine concentrations by liquid chromatographic-tandem mass spectrometry. Many participants had undetectable NDMA levels following $300 \mathrm{mg}$ ranitidine irrespective of dietary nitrate intake. There was no significant difference in 24 hour urinary excretion of NDMA with ranitidine $(0.6 \mathrm{ng})$, placebo $(10.5 \mathrm{ng})$, the non-cured meat diet (11.9 ng) or the cured meat diet $(23.4 \mathrm{ng})$. Similar findings were reported for DMA. This study is encouraging but it must be remembered that these were all healthy volunteers and did not have any known GI issues. Furthermore, even though diet was accounted for, the exact amounts of dietary nitrate, nitrite and NDMA were not quantified. This study is a first step to the return of a medication that many patients have relied on for many years.

\section{Gut microbial signature and immune response in non- alcoholic fatty liver disease (NAFLD) and hepatocellular carcinoma (HCC)}

Behary J, Amorim N, Jiang X, et al. Gut microbiota impact on the peripheral immune response in non-alcoholic fatty liver diseaserelated hepatocellular carcinoma. Nat Commun 2021; 12(1): 187.
The gut microbiota is altered in patients with NAFLD and HCC. These alterations were studied in three cohorts of patients: NAFLD-cirrhosis $(n=28)$, NAFLD-HCC $(n=32)$ and non-NAFLD control subjects $(n=30)$. Subjects with NAFLD-HCC and NAFLDcirrhosis were matched for severity of liver disease. Faecal samples were dysbiotic in the NAFLD-HCC and NAFLD-cirrhosis patients compared with controls, with reduction in $\alpha$-diversity indices, observed number of species and Chao-1-richness indices. There was expansion of Proteobacteria in the NAFLD-HCC cohort as opposed to controls $(p=0.041)$, but not with the NASH-cirrhosis group. Expansion of Enterobacteriaceae was observed in NAFLD-HCC compared with both NAFLD-cirrhosis $(\mathrm{p}=0.033)$ and non-NAFLD controls $(p=0.025)$. Several other alterations were also documented among the three groups at genus and species levels. Peripheral blood mononuclear cell samples were exposed to their individual bacterial extract to gauge the peripheral immune response. Subjects with NAFLD-HCC were shown to have a peripheral immunosuppressive profile compared with NAFLD-cirrhosis and non-NAFLD controls with increase in regulatory $T$ cells and reduction in cluster of differentiation $8+\mathrm{T}$ cells. This study highlights that gut dysbiosis and altered immune responses may act as cofactors in development of HCC in NAFLD subjects.

\section{REVIEWERS}

Dr Tom G Bird, University of Edinburgh, Royal Infirmary of Edinburgh, Edinburgh, UK.

Dr Christopher A Lamb, Newcastle University \& Newcastle upon Tyne Hospitals NHS Foundation Trust.

Dr Stuart McDonald, Centre for Tumour Biology, Barts Cancer Institute, London, UK.

Professor Raja Atreya, University Hospital Erlangen, Germany Dr John Leeds, Newcastle upon Tyne Hospitals NHS Foundation Trust, Newcastle Upon Tyne, UK.

Dr Ashis Mukhopadhya, Aberdeen Royal Infirmary, Aberdeen, UK.

\section{JOURNALS REVIEWED}

Science Translational Medicine, Nature, Science, New England Journal of Medicine, Journal of the American Medical Association and Nature Communications.

Funding The authors have not declared a specific grant for this research from any funding agency in the public, commercial or not-for-profit sectors.

Competing interests None declared.

Patient consent for publication Not required.

Provenance and peer review Not commissioned; internally peer reviewed.

(c) Author(s) (or their employer(s)) 2021. No commercial re-use. See rights and permissions. Published by BMJ.

\section{Check for updates}

To cite Smith PJ. Gut 2021;70:2004-2005.

doi:10.1136/gutjnl-2021-325934

ORCID iD

Philip J Smith http://orcid.org/0000-0003-1568-3978 